\title{
Turismo de Base Comunitária no Brasil. Insustentabilidade é uma Questão de Gestão
}

\author{
Community Based Tourism in Brazil. Unsustainability is a Matter of Management \\ Turismo Comunitario en Brasil. Insostenibilidad es una Cuestión de Gestión
}

Eduardo Jorge Costa Mielke ${ }^{1}$

Fernanda Vasconcellos Pegas ${ }^{2}$

\begin{abstract}
Resumo
O presente artigo analisa a questão do turismo de base comunitária (TBC) como metodologia de trabalho que almeja a melhora das condições de vida das comunidades que optam por esta atividade como estratégia. A medida em que o objetivo é discutir questões metodológicas do processo em desenvolvimento de projetos, como acesso a mercado, governança e monitoramento o trabalho apresenta estudos de caso brasileiros onde processo de cooperação e institucionalização de iniciativas através de associações e cooperativas de turismo foram vitais à sustentabilidade em todos os níveis. Em função da complexidade e das inúmeras variáveis que estão implícitas que estão atraladas à atividade turística em comunidades, o artigo também aponta possibilidades de avanço na adoção de estratégias locais ao envolvimento comunitário, onde o trabalho com a cadeia de valor do turismo no que tange a produção associada ao turismo pode mostrar melhores resultados.
\end{abstract}

Palavras-chave: turismo de base comunitária; desenvolvimento; governança; monitoramento.

\begin{abstract}
This article analyses community-based tourism (CBT) as a methodology of work that aims to improve the livelihood of the communities that choose this activity as an economic development strategy. Using examples from Brazil, the objective is to address methodological questions about the development stages as well as issues pertaining to access to markets, governance and monitoring. These case studies demonstrate that in order to achieve and maintain the sustainability of community-based tourism programs the presence of tourism associations and cooperatives that fomented initiatives of cooperation and institutionalization has been vital. Due to the complexity and existing variables that are both implicit and linked with tourism activities in communities, this article also provides suggestions to increase the

\footnotetext{
${ }^{1}$ Doutor em Desenvolvimento, Planejamento e Gestão do Turismo pela Universidade de Málaga, Espanha. Professor Adjunto do Departamento de Turismo da Universidade do Estado do Rio de Janeiro - UERJ. Brasil. Email: eduardomielke@yahoo.com.br.

${ }^{2}$ Pós-Doutorado no International Centre for Ecotourism Research - Griffith University - Gold Coast Campus. Austrália. E-mail: f.pegas@griffith.edu.au.
} 
adoption of local strategies that involve the community in tourism development and implementation, whereas working with local tourism chain can provide better results.

Keywords: community-based tourism; development; governance; monitoring.

\section{Resumen}

Este artículo analiza el tema de turismo de base comunitaria (por confirmar) como una metodología de trabajo que tiene como objetivo mejorar las condiciones de vida de las comunidades que optan por esta actividad como una estrategia de desarrollo local. En la medida en que el objetivo es examinar las cuestiones rasgos metodológicos tales como acceso a los mercados turísticos, la gobernanza y la evaluación y control de resultados y acciones, el presente trabajo presenta estudios de caso en Brasil, donde la cooperación y la institucionalización de las iniciativas a través de asociaciones y cooperativas son vitales para el turismo la sostenibilidad en todos los niveles. En función de la complejidad y las numerosas variables que están implícitas que se vinculadas al turismo en las comunidades, el artículo también señala las posibilidades de avance en la adopción de estrategias locales para la participación de la comunidad, donde el trabajo con la cadena de valor del turismo con respecto a la de producción relacionados con el turismo puede mostrar mejores resultados.

Palabras-clave: turismo de base comunitaria; desarrollo; gobernanza; monitoreo.

\section{Introdução}

Turismo de Base Comunitária ou TBC, não é um segmento, mas sim uma metodologia de trabalho. Desde que o turismo começou a ser usado estrategicamente como uma ferramenta para reduzir a pobreza na década de 80, dois processos foram iniciados quase simultaneamente: Ecoturismo e Turismo de Base Comunitária - TBC (The Mountain Institute, 2000; WWF International, 2001; United Nations Development Program - UNDP, 2006; United Nations World Tourism Organization - UNWTO, 2004, 2006; Caribbean Tourism Organization - CTO, 2007; Hainsworth \& Jamieson, 2007). A idéia era, e ainda é, a promoção do turismo envolvendo direta e indiretamente, todas as comunidades que residem ou estão muito perto de áreas de conservação (ex: parques nacionais). Muitas organizações têm trabalhado duro para isso. Durante esses anos, centenas de iniciativas têm sido realizadas na América Latina, muitas das quais são encontradas em todos os biomas brasileiros. 
Como em outros países do continente, a maioria dos projetos de TBC no Brasil enfrentam as mesmas situações-problemas que são divididos em três grupos. Há um consenso acerca dos processos que resultam neste insucesso: a) acesso ao mercado (Scheyvens, 2007; Epler Wood \& Jones, 2008), b) governança interna (Mitchell \& Muckosy, 2008; Santinni \& Harold, 2009) e c) gestão das parcerias estratégicas de interesse (Jordan, Hunt \& Stronza, 2008). A ineficácia de um ou mais destes processos faz com que mais de três quartos desses projetos de TBC sejam frágeis e/ou insustentáveis a longo prazo (Mielke, 2009). A maioria deles necessita de assistência externa constante. Cada um, por sua vez, está relacionado (todavia em graus diferentes) a todos os elementos de uma verdadeira cadeia de valor englobando desde doadores e financiadores, agências de fomento, setor público, organizações nãogovernamentais, e consultores, até empresas de turismo locais, os agentes intermediários de turismo (agências e operadoras turísticas) e evidentemente as próprias comunidades (Buckley, 2003; Stronza \& Pegas, 2008).

Aparentemente, a falta de uma metodologia para implementar adequadamente um projeto de turismo comunitário para o correto encadeamento deste processo se faz necessária. Instrumento este que possa ser aplicado independentemente de distintos contextos socioeconômicos e históricos, pois de acordo com Mitchell \& Muckosy (2008), a questão chave é a organização e estrutura comunitária que influenciam na forma com que as comunidades comercializam seus produtos e serviços turísticos como também a distribuição/uso dos lucros.

$\mathrm{Na}$ América Latina pode-se contar com bons exemplos, embora bem pontuais, onde a atividade turística pode contribuir com a melhora das condições de vida (Stronza \& Gondillo, 2008; Epler Wood \& Jones, 2008; Pegas \& Stronza, 2010). Num país continental de 8,5 milhões de $\mathrm{km}^{2}$, o grande desafio brasileiro é precisamente planejar um programa de governo, em âmbito nacional de desenvolvimento turístico, executando-o de forma a atender a diversidade sóciocultural e ambiental presente no país. Não obstante, no Brasil pode-se encontrar alguns casos de sucesso que mostram que é possível alcançar resultados, desde que aspectos metodológicos estritamente ligados a questão da governança comunitária e acesso ao mercado turístico sejam observados desde o início do processo metodológico (aplicação do projeto de $\mathrm{TBC}$ ). Questões históricas, culturais e sociais da comunidade são importantes e devem ser consideradas, porém deixam de ser tidas como limitantes, na medida em que a 
questão central é a gestão turística comunitária (Mielke, 2009; Pegas \& Stronza, 2010; Rodriguez, 2008).

Além desta introdução e conclusão, este artigo está dividido em três seções. Na primeira, será debatido como e por que tais aspectos supracitados têm influenciado os resultados atuais dos projetos de desenvolvimento de TBC no Brasil, relacionando-as com o papel e as responsabilidades de cada elemento da cadeia. Ainda nesta seção serão discutidos aspectos importantes referentes aos processos de monitoramento e avaliação de projetos de TBC. Na seção seguinte objetiva-se fornecer soluções oportunas e possibilidades de modo que será possível enfrentar os desafios atuais, citando três exemplos em que tais processos foram realizados de forma estratégica voltada para resultados mensuráveis. Por fim, de forma introdutória, discute-se a questão do monitoramento de resultados aplicado aos projetos de TBC.

\section{A Insustentabilidade de Projetos de TBC}

Infelizmente o insucesso de projetos de TBC é relatado por vários autores (ex: Ashley et al, 2001; Caribbean Natural Resources Institute - CANARI, 2004; Hitchens and Highstead, 2005; Dixey, 2008). Uma pesquisa levada a cabo pela Rainforest Alliance and Conservation International em 200 projetos analisados em todo o continente americano revelou que a taxa média de ocupação de meios de hospedagem gerenciados por comunidades foi de 5\%. Para os mesmos autores "o mais provável resultado para uma iniciativa em TBC é que o projeto seja insustentável após o término do período de financiamento" (Mitchell \& Muckosy, 2008 p. 2). Este resultado advém por problemas e/ou falta de processos sustentáveis de gestão turística envolvendo governança e acesso ao mercado. Este discurso é comum sendo que alguns casos são utilizados como argumento para gerar esforços para captação de mais recursos. Mas porque esta realidade é tão presente? Por que boa parte dos projetos chega ao final com poucos resultados tangíveis? Ou melhor, por que o sucesso é ainda a exceção à regra?

É fundamental deixar muito claro que os projetos de turismo de base comunitária devem ter como princípio básico a melhora da condição de vidas das pessoas que residem em regiões onde, via de regra, são excluídas do sistema econômico. Regiões estas onde o estado se faz pouco presente e uma das poucas possibilidades de incremento de renda e geração de 
emprego se dá através justamente de iniciativas onde a atividade econômica de fundo é o turismo. Este também é o caso de áreas em que são as comunidades quem são donas das terras em que o turismo é implementado, exemplo são as comunidades indígenas que usam o TBC como base para o ecoturismo. Nestes casos, o turismo pode vir a ser uma ferramenta para gerar alternativas de renda como também benefícios diretos para a conservação de biodiversidade - quesito chave em projetos de ecoturismo. Esta provavelmente é a razão pela qual tais projetos já iniciam com uma grande expectativa com relação aos seus próprios desdobramentos.

Um aspecto deve ficar extremamente claro: projetos de turismo comunitário devem ter uma concepção filosófica dentro dos preceitos do desenvolvimento econômico. Do ponto de vista teórico, as linhas de pensamento que envolvem as discussões do desenvolvimento endógeno, de baixo para cima e processos de aglomeração como os clusters, arranjos e sistemas produtivos locais são leituras obrigatórias para o entendimento do que vem a ser os pilares da sustentabilidade do TBC (Amaral, 1995, 2002; Barbosa \& Zamboni, 2000; Esteve, 2002; Garrido, 2001; Dreher et al, 2006; Cassiolato \& Szapiro, 2003; Cassiolato et al, 2005; Mielke \& Pereira, 2006; Jamal \& Stronza, 2009). Isto porque é fundamental que, em linhas gerais, boa parte destes temas aborda com profundidade um ponto fundamental, que são os processos de cooperação.

Dentro desta mesma linha, o trabalho envolvendo a atividade turística é complexo e dinâmico, pois envolve uma série de atores sociais locais (comunidades, empresas turísticas - ou não, organizações governamentais - ou não) e não locais como os turistas e intermediários, bem como suas inter-relações (Mielke \& Pereira, 2006). Sob a perspectiva do negócio em si, TBC não difere em nada de qualquer outra relação comercial: algo se vende e se compra criandose, portanto, as expectativas entorno do serviço vendido. Esta troca por sua vez deve ser feita com responsabilidade e qualidade. Deve gerar margem de lucro adequada para a manutenção e crescimento (equilibrado) dos negócios individuais de quem diretamente está envolvido e ser percebido por aqueles que indiretamente estão participando deste processo. Os projetos de TBC também devem gerar um lucro suficiente para sustentar as iniciatias turísticas como também os projetos vinculados a melhoria da qualidade de vida dos moradores locais. Atividades, estas, que são tanto de curto como longo prazo e devem ser discutidas e inseridas no plano de manejo e implementação do TCB em todas as suas fases. 
Todo este processo tem custos, onde a remuneração deve acontecer e ser percebida coletivamente e claramente discutida antes da implementação e desenvolvimento das atividades de turismo. Em outras palavras se o projeto de TBC não contribuir com o aumento dos rendimentos das famílias no médio prazo - até 12 meses de sua implementação (Mielke, 2010) - o mesmo perderá a confiança da comunidade e terá sua sustentabilidade política e econômica comprometidas. Existe também o risco de danos ambientais em virtude desta limitação. Neste caso, tal impacto pode causar sérios danos a viabilidade do turismo local principalmente se as atividades promovidas são as do ecoturismo. Contudo, o quanto deve ser este aumento $(\%)$ e ou como isto resultará no processo de tomada de decisão, na liderança e na divisão do poder e no empoderamento da comunidade, é o que torna cada projeto singular.

Antes de analisarmos as situações-problema e de como elas podem ser trabalhadas é importante estabelecer alguns aspectos de análise. Todas elas estão intimamente relacionadas entre si dentro do processo metodológico que vai levar um grupo de pessoas ou comunitários ao sucesso ou fracasso. É importante destacar que inicitivas de TBC devem ser consebidas no longo prazo. Não há como estruturar uma ou mais comunidades, preparando-as para lidar com as situações-problema e identificar oportunidades em um período inferior a 18-24 meses. Isto é fácil entender sob a perspectiva do negócio turístico. $\mathrm{O}$ acesso ao mercado torna-se um problema quando as relações com os canais de distribuição não estão estabelecidas. Ou, não está claro ou não há recursos para saber onde está e como atrair o público de interesse.

Isto ocorre, por sua vez, pois a comunidade não foi preparada adequadamente para lidar com as relações entre ela mesma e das organizações turísticas dentro da sua realidade. Em outras palavras, muito antes de ter resultados financeiros, o que também dita/indica o sucesso está na harmonia e equilíbrio do contexto social-institucional das comunidades envolvidas. Nestes casos, o que prevalece é uma limitação de um processo consolidado de preparação e formação da liderança e dum processo de tomada de decisão que tenha sido legitimado pela comunidade como um todo. Isto diz respeito a Governança Interna que não proporciona o desenvolvimento de um fluxo democrático e estratégico da informação. Sendo assim, não está claro o que de fato o turismo aporta ou pode aportar, qual são os pros e contras, e/ou qual é saldo da relação custo-benefício. Por exemplo, no estudo não publicado desenvolvido pelo Departamento de Turismo da Universidade Estadual do Rio de Janeiro em 2011 em parceria com Ministério do Turismo do Brasil em 2010, revelou que mais de $70 \%$ das comunidades 
dos 36 projetos analisados em todo o país que obtiveram recursos do Ministério $(\sim \mathrm{R} \$ 150$ mil/cada) para desenvolvimento do TBC entre 2008 e 2011 não entende o papel do turismo como instrumento de geração de renda e emprego. Sem foco e conhecimento do que esta atividade representa e/ou pode representar, sugere-se que torna-se muito difícil eleger o que é ou não é prioridade para a própria comunidade.

E, por fim, sem este processo estando claro não se desenvolve o ambiente necessário a construção de processos cooperativos, colaborativos e construtivos entre a comunidade interna com suas representações e o público de interesse externo. Não há confiança em saber se expectativas serão de fato atendidas envolvendo o pós-venda. Num mercado competitivo em que o número de possibilidades de escolha das férias se prolifera a cada dia, ter certeza da garantia de satisfação é vital. Se a comunidade não tem um conhecimento explícito do que vem a ser turismo, seus benefícios e riscos, e ter uma visão mais clara de suas metas, objetivos e limites, fica muito difícil para esta saber elencar quem são as fontes (ex: empresas e operatoras turísticas) que irão trazer um perfil de turista que interessa e pode ser implementado pela comunidade e que promoverá um comportamento e um gasto responsável e não-sazonal. Por esta razão a importância do papel das ONGs, as quais a presença é quase que essencial em iniciativas de TBC, desde que estas conheçam, por sua vez, a dinâmica turística no contexto do turismo comunitário.

\section{Acesso ao Mercado, Governança Interna e Gestão das Parcerias Estratégicas de Interesse}

O baixo acesso ao mercado é umas questões principais pelas quais os projetos TBC não tornam-se sustentáveis após o período de investimento. Isto acontece porque ou as comunidades oferecem produtos de baixa qualidade ou estabelecem de maneira tardia as relações comerciais com os intermediários. Em muitos casos, há uma certa aversão em se trabalhar com os mesmos. As comunidades de uma forma geral observam com muita reticência o ato de negociar com agências e operadoras turísticas. No imaginário, há uma forte imagem do papel explorador e não de colaborador com que as relações entre comunidades e intermediários se estabelecem. O papel destes agentes no contexto do desenvolvimento turístico de base comunitária é, todavia, fundamental e deve ser estabelecido no momento em que as iniciativas são concebidas. São eles que detém o conhecimento e podem dar o 
direcionamento de produtos turísticos a serem estruturados dentro da comunidade, alinhados com padrões desejáveis de qualidade sócio-econômico e ambiental. Contudo há critérios para serem observados. Primeiro, no Brasil há centenas de operadoras turísticas e dentro deste grupo deve identificar-se aquelas que possuem a responsabilidade socioambiental como princípio pois cada vez mais este tema ganha força e influência no mercado do turismo nacional e internacional (Buckley \& Pegas, 2011). Buscar tais agentes quando o projeto está nas fases finais é um erro fatal.

Outro aspecto importante é que nem sempre o desenvolvimento turístico ocorre de forma direta onde a comunidade recebe turistas. Em muitos casos, devido a complexidade e estágio de imaturidade das comunidades em desenvolver a atividade turística, torna-se mais simples, viável, e fácil, em termos de resultados operacionais, o desenvolvimento de ações indiretas. Trata-se daquelas onde o trabalho se dá na leitura, mapeamento da cadeia de valor do turismo local e otimização da produção agropecuária já levada a cabo tradicionalmente pelas comunidades. Nestes casos o desenvolvimento de projetos de produção associada ao turismo local tem sido uma estratégia importante na inclusão social das comunidades localizadas próximas a destinos turísticos.

Como uma mediação equilibrada é possível estabelecer outro padrão de produção organizada e comercialização da produção rural reestruturando a cadeia de fornecedores das empresas turísticas. Além de proporcionar ganhos dentro da dinâmica laboral das comunidades, este processo tambem reduz a probabilidade dos impactos sociais oriudos da visitação turística. Trabalhando com comunidades Quilombolas na Bahia foi possível organizar a produção de frango e verduras comercializando-a diretamente com hotéis da região que em contrapartida credenciam tais alimentos dentro da política socioambiental, oferencendo aos hóspedes alimentos orgânicos, além de comprarem alimentos mais baratos. Este tipo de ponte direta entre a comunidade e empreendimentos turísticos também tem sido fomentada na comunidade indígena de Infierno, Peru. Como parte das iniciativas do TBC, membros da comunidade vendem produtos etno-botânicos e artesanato aos turistas que se hospedam no ecolodge (Jordan, Hunt \& Stronza, 2008). Além de oferecer uma alternativa de renda, esta iniciativa também oferece uma opção para as famílias que não querem estar diretamente em contato com os turistas mas, ao mesmo tempo, ter um benefício econômico. 
Em outros casos, há um mix de possibilidades consorciando comunitários que possuem estrutura receptiva para turistas e que ao mesmo tempo possuem outros comunitários na sua cadeia de fornecedores. Este exemplo é observado em várias inicitivas organzidadas como cooperativas e associações de turismo. Em ambos os modelos produtores rurais ou propriedades que possuem atrativos como orquidários, participam do turismo local fazendo parte direta e indiretamente do produto turístico local. Trata-se de um processo coloborativo de resultados onde em linhas gerais os produtores passam a diversificar os canais de venda utilizando-se das empresas locais onde o investimento na construção destas parcerias não é alto. Estas atividades são comuns nas áreas promovidas como "rotas de turismo rural" onde iniciativas individuais favorecem e beneficiam o potencial turístico do conjunto. Estes resultados também podem ser obtidos a nível local, em que vários participantes da mesma comunidade contribuam, e assim se beneficiem, para com os serviços e recursos associados as atividades turísticas (Trejos \& Chiang, 2009; Saxena, 2006). Tais sinergias podem, assim, fomentar o desenvolvimento de sentimentos de confiança e identidade local.

A Cooptur, com sede na cidade de Ponta Grossa (120 km de Curitiba, capital do Estado do Paraná, Região Sul) é a primeira cooperativa brasileira formada por empresários turísticos de sete cidades diferentes. Desde 2004, seus cooperados estão comprometidos com a qualidade e estruturada oferta turística tendo o produtos e serviços de aventura aliados a treinamentos corporativos, direcionados a empresas de diversos setores da economia estadual interessadas em capacitar seus empregados. O grande diferencial foi buscar nas parcerias estratégicas a sustentabilidade financeira e a ampliação das suas ações.

Fruto deste modelo estruturou-se em 2006 a segunda cooperativa, a Cooperguará-Ecotur. Localizada no litoral do Estado, a entidade se fortalece na cooperação de seus membros para a resolução de problemas como os da sazonalidade que prejudica a renda das familias na baixa temporada. Ao longo do processo de estruturação da cooperativa um forte plano de negócios foi elaborado em conjunto com a comunidade. A partir deste documento foi possível verificar e agir quanto aos gargalos e necessidades ao desenvolvimento da coopertiva. O grupo cooperado teve na construção de sólidas relações com o mercado e na hospitalidade dos comunitários a base para sua sustentabilidade. Ambas empresas sociais são atuantes e informações podem ser obtidas em www.cooptur.coop.br e www.visiteguaraquecaba.com.br. 
Saber negociar parte do pressuposto que o grupo comunitário tenha voz (Stronza \& Pegas, 2008; Mielke, 2009). Estruturar este processo no âmbito das comunidades é uma tarefa complexa. Isto é devido a dois aspectos fundamentais. Primeiro, pois na composição de um produto turístico há o envolvimento de uma série de empresas turísticas, tais como: transportes, meios de hospedagem, guias, bares e restaurantes. Além dos atrativos da região. Do ponto de vista administrativo cada uma destas empresas possui uma base de custo/receita diferente, logo seus proprietários possuem interesses, visões, e requerimentos/necessidades distintas do negócio turístico. Via de regra, aumentar o preço das pousadas pode reduzir o fluxo turístico o que irá afetar diretamente o fluxo de caixa das empresas de transportes e/ou o ganho dos condutores que cobram por turista. Transformar esta diversidade em um modelo de gestão coletiva (cooperativas ou associações de turismo) que possibilite ganhos coletivos e sobretudo individuais de forma equilibrada é um desafio que deve ser ultrapassado. E em segundo lugar, trata-se do perfil da região que indicará estrategicamente quais são as possibilidades de mercado onde há maior apelo. Ou seja, cada região tem um ou dois segmentos dos quais seu perfil ou vocação turística está mais adequado e onde muito provavelmente a oferta turística local e regional estará alicerçada direcionando assim seus esforços para otimizar uma certa identidade. Ocorre que todo processo de desenvolvimento turístico pode ser limitado quando por ventura empresas turísticas prestam serviços não alinhados a este processo de segmentação.

Um exemplo a ser citado no contexto desta seção é a inicitiva Acolhida na Colônia. Criada no Brasil em 1998 proporciona que agricultores hospedem turistas proporcionando troca de experiências entre pessoas. Sob uma base jurídica de associação, conta com uma central de reservas e informações. O modelo importado, adaptado e aplicado no Estado de Santa Catarina e dentro da realidade brasileira possibilita a inserção do modo de vida rural consorciando com a atividade turística. Essencialmente dentro do conceito de base comunitária, a iniciativa traz geração de renda e emprego direto à região de atuação, onde cada integrante possui seus deveres e responsabilidades perante o grupo.

Outro exemplo é o caso da Reserva de Desenvolvimento Sustentável de Mamirauá, no Estado do Amazonas. Desde 1999, o Instituto de Desenvolvimento Sustentável Mamirauá (IDSM) implanta diferentes iniciativas voltadas a iniciativas que fomentem desenvolvimento econômico sustentável vinculado a capacitação social e conservação ambiental (ex: ecoturismo, 
educação ambiental, manejo florestal e pesqueiro) (Arantes, 2009; Queiroz \& Peralta, 2006; IDSM, 2011). Turismo é implantado no formato de TBC usando ecoturismo como viés para conservação e desenvolvimento econômico para com sete comunidades ribeirinhas. Como no caso de Santa Catarina, as iniciativas do turismo têm gerado benefícios econômicos como também benefícios a nível social e ambiental. $\mathrm{O}$ uso destes, como também os planos de manejo, são elaborados, regulamentados e acompanhados pelas comunidades envolvidas (IDSM, 2011).

Em linhas gerais todo processo de construção de uma governança interna sólida inevitavelmente passa pelo processo de institucionalização comomunitário. Trata-se da constituição de empresas sociais com as quais as decisões passam a valer no papel. Devido a diversidade e a não frequência de processos semelhantes no ambiente comunitário, a elaboração de instrumentos normativos abre campo para o desenvolvimento das responsabilidades e deveres dentro do grupo social. Como a atividade turística exige qualidade na prestação dos serviços, o estabelecimento de regras coletivas é fundamental. Para tanto, está pressuposto que o comprometimento exista, bem como processos de cooperação sejam práticas a serem desenvolvidas de forma cada vez mais frequente ao longo da execução de um projeto de TBC.

De acordo com Boesche (2005) e Mielke (2009), um dos instrumentos capazes de medir este processo é a elaboração e discussão do Regimento Interno e Estatuto Social mesmo para iniciativas onde a organização esteja ainda sendo discutida. No estudo supracitado realizado no Brasil em 2011, dos 36 projetos estudados, somente 3 deles contaram com alguma presença de agentes de mercado (intermediários) no processo de discussão e elaboração. Sendo que somente em 1 há contrato formal entre comunidade e mercado.

$\mathrm{Na}$ medida em que a governança se estabelece fica cada vez mais claro o estabelecimento de metas e objetivos mais claros. Reduzem-se também as dúvidas pelas quais muitos comunitários observam a atividade turística. Torna-se possível planejar de forma mais estruturada, bem como buscar nas organizações governamentais e não-governamentais parcerias estratégicas ao desenvolvimento de ações de fomento e/ou melhoria da qualidade turística local. Contudo é importante observar que a presença e a escolha de organizações de interesse devem seguir critérios estabelecidos pela comunidade com base na compreensão do papel da atividade turística e seus impactos. Assim como intermediários, ao longo do processo 
de construção do grupo comunitário para o desenvolvimento e gestão do turismo há uma série de entidades que podem e devem ser envolvidas.

\section{Monitoramento e Avaliação de Projetos de TBC}

Infelizmente, o monitoramento de processos e resultados dentro de um programa de avaliação em projetos de TBC não tem sido uma prática corriqueira (Dixey, 2008; Santinni \& Godwin, 2009). E, quando ela é feita, ocorre de maneira geral dentro de dois percausos. Primeiro, trata-se dos critérios ou indicadores utilizados. Via de regra, as próprias organizações que captam os recursos para um determinado projeto possui seus próprios critérios que serão apresentados aos públicos de interesse, dentre eles as próprias instituições doadoras destes recursos, ferindo o princípio da imparcialidade. Este aspecto é importante, pois dentro do contexto da responsabilidade no turismo, doadores deveriam ser mais presentes ao longo do processo de monitoramento.

Também existem casos em que entidades doadoras estipulam regras como condição para o apoio financeiro, como a inclusão de minorias e mulheres, transparência da distribuição de benefícios e no desenvolvimento das atividades turísticas (Jordan, Hunt \& Stronza, 2008; IDSM, 2011). Dependendo da estrutura sociocultural e contexto monetário das comunidades, principalmente no caso das comunidades indígenas, estas novas regras podem criar disarmonia e/ou quebra de tradições, o que pode dificultar a implementação das iniciativas pré-estipuladas e/ou desejadas (Leach, Mearns, \& Scoones, 1999; Rodriguez, 2008).

Doar sem controle é investir cegamente e o prejuízo pode ser muito maior do que o recurso perdido. Por exemplo, caso as expectativas prometidas não sejam cumpridas pode haver um impacto negativo na auto-estima de pessoas envolvidas pois estas muitas vezes vivem em situações socioeconômicas de pouca oportunidade ou economicamente frágeis. Para SNV\&HU (2008: 8) "O monitoramento é o processo de mensuração normalmente feita através de indicadores, a fim de proporcionar uma melhor compreensão da situação atual, bem como alguma idéia das tendências de desempenho". Trata-se de um processo de responsabilidade de todos e que deve ser compartilhado (Claude, 2000).

Outro fator diz respeito ao próprio processo de monitoramento interno das atividades para que estas atingam as metas traçadas. Primeiro, pois a ineficiência financeira de muitos projetos de 
TBC pode também estar ligada a falta de capacitação interna dos moradores locais, o que sustenta a dependência destes - ou da comunidade em geral - ao apoio financeiro e assistência adminstrativa oferecida pelos orgãos públicos e ONGs (Buckley, 2003). As iniciativas de ecoturismo nas comunidades de Infierno no Peru (Stronza \& Pegas, 2008), Praia do Forte, Brasil (Pegas, Coghlan \& Rocha, 2011) e na Reserva de Desenvolvimento Sustentável de Mamirauá, Brazil (IDSM, 2011) são exemplos em que a capacitação local acontece em paralelo com a implementação do ecoturismo.

E em segundo lugar, atualmente não há um consenso geral acerca de quais seriam estes critérios a serem monitorados para saber ou antever se um projeto de TBC poderá dar ou não certo (Amorim, Dias \& Mielke, 2008). Contudo, estabelecer indicadores específicos a serem aplicados a todos os casos não é totalmente recomendável. Isto porque, como estamos lidando com pessoas, culturas regionais que determinam compartamentos distintos, metodologias de trabalho devem ter o cuidado à adaptação local.

No caso brasileiro, os critérios de concepção, metas e prazos de aplicação de um projeto de TBC nas Regiões Norte e Nordeste do país são totalmente diferentes com características, traços histórico-culturais e atribuitos nas Regões Sul ou Sudeste. Por exemplo o próprio papel do Estado no entendimento das pessoas que vivem em diferentes regiões do país divergem drasticamente (Almeida, 2007). Outros fatores diferenciais referem-se a colonização (ex: base na escravatura e sistema parternalista versus imigração de colonos europeus) e articulações colaborativas entre as comunidades rurais (ex: Pegas \& Stronza, 2010; Pádua, 1999). Para a Brooks World Poverty Institute (BWPI) (2009) muito pouco tem se dado atenção as metodologias de análise de programas de monitoramento e avaliação, sendo que "o monitoramento traz em tempo real informações que a evolução dos processos do projeto como um todo" (BWPI 2009, p. 5).

Sprecher \& Jamieson, 2000; Vellas 2005; Mielke \& Sperandio, 2009 destacam que muitos dos indicadores deste processo estão baseados segundo critérios quantitativos, como por exemplo, taxa de ocupação e/ou fluxo turístico, que estão baseados em cenários pouco realísticos (Santinni \& Goodwin, 2009). Maris \& Schipani (2001) e o World Bank (2006) destacam a importância do uso de critérios qualitativos, como por exemplo, nível de cooperação, entendimento da dinâmica turística, equilíbrio do relacionamento com demais públicos de interesse, liderança, envolvimento das mulheres e processo de tomada de decisão. 
Entretanto, a grande maioria dos documentos analisados traz e discute indicadores quantitativos em detrimento aos qualitativos. Reidar (2004) destaca a importância de um mix metodológico e sua retroalimentação possibilitando uma constante observação quanto aos objetivos das ações pretendidas do projeto. (Jha et al., 2007; Kane \& Trochim, 2007; Ashley \& Mitchell, 2008; Morra \& Rist, 2009; The Global Fund, 2010). De forma prática, tais critérios os mesmos fazem referência a indicadores relativos aos processos de cooperação com que as comunidades desenvolvem entre os próprios membros e destes com os públicos de interesse - poder público, agências não governamentais e sobretudo com as empresas turísticas locais e intermediários (Mielke \& Nascimento, 2009; Godfrey \& Clarke, 2000; Wearing \& McDonal, 2002).

Além disso, é essencial analisar o comprometimento participação (Bond et al., 2001; Chapman \& Kirk, 2001; Jones, 2005; Bray et al., 2005; Sebola \& Fourie, 2006) e dinâmica de poder no que tange a organização comunitária (Wilson et al, 2001; Laverack \& Thangphet, 2007; Maclellan et al., 2007), a liderança e tomada de decisão (Williams \& Wade, 2002; Ife, 2002; Kirk \& Kraft, 2004; Mills, 2005; Moscardo, 2008), presença de Estatutos e Regimentos Internos para grupos organizados (Boershe, 2005; Mielke, 2010). Porém, se boa parte das comunidades pesquisadas não tem claro como o turismo pode proporcionar o desenvolvimento de oportunidades de geração de renda e emprego, logo se torna desafiante: a) estabelecer metas e processos relativos a tais critérios qualitativos e b) identificar como os mesmos irão influenciar a dinâmica de execução de projetos. Mielke (2010) sugere que tais indicadores quantitativos devem fazer parte do processo metodológico como metas de organização comunitária, sendo acompanhados desde o início das primeiras ações de qualquer projeto de TBC. A ausência destes indicadores comprometerá o estabelecimento de uma equilibrada cadeia de valor local do turismo, em consequiência comprometerá os resultados quantitativos do projeto.

\section{Conclusão}

O investimento e interesse por projetos envolvendo comunidades e turismo mostra a importância quanto ao desenvolvimento deste processo. Turismo de Base Comunitária (TBC) é um trabalho complexo e de longo prazo. Deve atender aspectos metodológicos que possam cumprir uma meta central: melhoria da qualidade de vida das pessoas diretamente envolvidas. 
Tais processos possuem exemplos nacionais, ou seja, disponíveis à sua replicabilidade.

É importante realizar que TBC é um negócio onde compra e venda de serviços e produtos que vai além do enquadramento como segmento. Trata-se de um instrumento de combate a pobreza e capacitação social através do desenvolvimento de relações comerciais envolvendo a atividade turística. Sendo que não necessariamente as comunidades devem receber turistas, como ocorre em alguns casos em que membros da comunidade têm contato direto com visitantes na prestação de serviços e/ou na intermediação de venda de produtos, como por exemplo, artesanato. O preparo das comunidades e o reforço nas relações de cooperação para a obtenção de resultados no incremento de renda e oportunidades de trabalho são premissas do processo de estabelecimento da governança que possibilite o entendimento das reais possibilidades de acesso ao mercado.

A comercialização do turismo é desenvolvida na medida em que se tem um profundo conhecimento acerca do perfil da relação oferta-demanda aplicado em cada Experiência. Cooperar no âmbito da atividade turística é lidar com um negócio com ganhos individuais e coletivos. Estabelecer suas regras e processos é um desafio constante.

No que tange o monitoramento de projetos de TBC, os critérios utilizados para tal são excessivamente quantitativos. Está claro que questões ligadas à qualidade tanto das relações entre comunitários entre si e destes com públicos de interesse, são à base de sustentação de projetos de TBC. E também parte porque as próprias comunidades não têm claro o papel do turismo em suas realidades. Há, todavia, uma expectativa não concretizada de como o turismo irá contribuir, impactar, e, fundamentalmente de como ele funciona.

No Brasil, há pouca inserção de intermediários turísticos ao longo processos verificados das Experiências, bem como baixa utilização de metodologias de planejamento de economia social, como por exemplo: Plano de Negócios, Estatutos e Regimentos Internos. A falta destes, em linhas gerais, indica pouco amadurecimento do debate entorno das responsabilidades, obrigações, direitos e deveres das pessoas diretamente e indiretamente envolvidas nos projetos. Recomenda-se em próximas ações maior trabalho direcionado acerca dos indicadores qualitativos no que tange sua inclusão na metodologia de concepção, elaboração e execução de projeto de TBC. Uma vez estabelecidas, torna-se possível debater metas dos projetos - variáveis quantitativas, otimizando e fundamentando o acesso ao mercado dos produtos turísticos comunitários. 
Os processos de Monitoramento devem ser iniciados junto com as primeiras ações do projeto. Trata-se do acompanhamento tendo como base, indicadores qualitativos estruturando um ambiente onde a gestão e a cooperação entre micro e pequenos empresários podem organizar a atividade em consonância ao perfil turístico local. $\mathrm{O}$ fortalecimento de processos de governança sólidos que possibilitem o desenvolvimento da autonomia na tomada de decisão deve ser observado ao longo de todo o processo de formação e solidificação do grupo de pessoas envolvidas.

Do ponto de vista estrutural e estratégico, percebe-se que há uma baixa valorização quanto a utilização dos recursos e a inserção da produção das comunidades na economia local. Pouco valor se dá a ela e a comercialização agrícola em detrimento ao que possivelmente a atividade turística pode proporcionar. Em um ambiente onde a atividade turística não é bem compreendida e a agricultura é a principal atividade de fato econômica, sugere-se um direcionamento ao trabalho de otimização da produção e inserção da produção como parte da cadeia de valor do turismo local.

Em muitos projetos recomenda-se direcionar o foco das ações entorno da Cadeia de Valor do turismo local. Isto porque além de trabalhar com atividades econômicas já assimiladas pelas comunidades e que estão dentro do seu entendimento. Este processo reduz os impactos sociais diretos do turismo. O objetivo é trabalhar dimensionando a cadeia de fornecedores das empresas turísticas locais com produtos local oriundos das comunidades.

\section{Referências}

ALMEIDA, A.C. A cabeça do brasileiro. Record, Rio de Janeiro, 2007.

AMARAL FILHO, J. Desenvolvimento regional endógeno: (re) construção de um conceito, reformulação de estratégias alternativas (à guerra fiscal). In Anais do XXIII Encontro Nacional de Economia. Salvador, Anpec. 1995.

É o negócio ser pequeno, mas em grupo. Fortaleza. [s.n], 1995.

AMORIM, B., Dias, A., Mielke, E. J. C. Análise de oito metodologias de Indicadores de sustentabilidade para projetos de desenvolvimento turístico. In: X SIT - Seminário Internacional de Turismo, 2008, Curitiba. X SIT - Seminário Internacional de Turismo, 2008.

ARANTES, C.C. Ecologia do pirarucu Arapaima gigas (Schinz, 1822) na várzea da Reserva de Desenvolvimento Sustentável Mamirauá, Amazonas, Brasil. Universidade Estadual de Santa Cruz - UESC. Dissertação de Mestrado. (2008). Acessado 18 Jan. Disponível em: <http://www.iieb.org.br/enviados/publicador_pdf/trab_Dissert_Caroline_Arantes.pdf.>. 
ASHLEY, C \& Mitchell, J (2008) Doing the right thing approximately not the wrong thing precisely: Challenges of monitoring impacts of pro-poor interventions in tourism value chains. London. Overseas Development Institute. Working paper 291. Accessado em 18 de fev. Disponível em: <http://www.odi.org.uk/resources/download/1590.pdf>.

BARBOSA, M. A C.; Zamboni, R. A Formação de um "cluster" em torno do turismo de natureza sustentável em Bonito - MS Brasília: IPEA (2000). (Texto para Discussão n 772). Mimeografado.

BERCHES, F. Community-based conservation in a globalized world. PNAS 104(39): 15188-18193, 2007.

BOESCHE, L. Fidelidade cooperativa:uma abordagem prática. Sescoop-PR. Curitiba, Brazil, 2005.

BOND, R., Curran, J., Kirkpatrick, C., Lee, N., and Francis, P. Integrated Impact Assessment for Sustainable Development: A Case Study Approach. World Development 29:1011-1024., 2001.

BRAY, D., Cornejo, M., Cohan, S., and Beitl, C. Community-based Ecotourism: A Conservation and Development Strategy in Search of an Analytic Framework. Unpublished paper. Annual Meeting of the Society for Applied Anthropology, Santa Fe, New Mexico, 2005.

BROOKS World Poverty Institute - BWPI. Using Mixed Methods in Monitoring and Evaluation: Experiences from International Development. BWPI Working Paper 107, Manchester, Reino Unido, 2009.

BUCKEY, R. Case studies in ecotourism. Cabi International: Oxfordshire, Reino Unido. 2003.

BUCLEY, R.C. and Pegas, FTourism and corporate social responsibility. Em: Holden, A. e Fennell, D. (eds) Handbook of Tourism and Environment. Routledge., 2011.

CASSIOLATO, J. E.; Lastres, H. M. M., Arroio, A. C. Sistema de Inovação e Desenvolvimento: Mitos e Realidade da Economia do Conhecimento Global. In: Helena Maria Martins Lastres; Jose Eduardo Cassiolato; Ana Arroio. (Org.). Conhecimento, Sistemas de Inovação e Desenvolvimento. Rio de Janeiro: UFRJ e Contraponto, p. 17-50., 2005.

CASSIOLATO, J. E.; Szapiro, M. H. S. Uma caracterização de arranjos produtivos e inovativos locais de micro e pequenas empresas. In: Helena M. M. Lastres, José E. Cassiolato e Maria L. Maciel. (Org.). Pequena empresa: cooperação e desenvolvimento local. Rio de Janeiro: Relume Dumará, p. 35-50.. 2003.

CHAPMAN, M., \& Kirk, K. Lessons for Community Capacity Building: A summary of the research evidence., 2001. Acessado em 18 de Jan. Disponível em:http://www.scothomes.gov.uk/pdfs/pubs/260.pdf.

CLAUDE, B. Performance-based management: case study on the monitoring and evaluation unit of phase 2, US Agency for International Development USAID, Washington, 2000.

CTO- Caribbean Tourism Organisation (2006) Competing with the Best: Good Practices in Community Based Tourism in the Caribbean [on line] [Accessed 12 February 2011]. Available from: http://www.onecaribbean.org/goodpractices/bestpractices.aspx>

DIXEY, L. The Unsustainability of Community Tourism Donors Projects: Lessons from Zambia. In. Responsible Tourism: Critical issues for Conservation and Development. Earthscan, London 323 342, 2008.

DREHER, M T., Oliveira, R A., Scmitdt, C M. Governança em APLs de Turismo: A Experiência da Aglomeração Empresarial situadana Região Rural da Nova Rússia em Blumenau, SC. IV SeminTUR Anais do Seminário de Pesquisa em Turismo do MERCOSUL Universidade de Caxias do Sul.,2006. 
EPLER Wood, M., Jones, H Community-based Tourism Enterprise in Latin America. Burlington. EplerWood International., 2008. Acessado em 18 de Jan. Disponível em: <http://www.eplerwood.com/pdf/Community_Based_Tourism_Enterprise.pdf>

ESTEVE Secall, R.. Los agrupamientos (clusters) turísticos como base analítica de Ia competitividad de los territórios de orientación turística. Málaga, Universidad de Málaga, 2002. Mimeografado.

GARRIDO, I. M. D. A. Modelos multiorganizacionais no turismo: cadeias, clusters e redes. 124 p. Escola de Administração, Universidade Federal da Bahia, Salvador. 2001. Dissertação de Mestrado não publicada.

GODFREY, K., \& Clarke, J. The tourism development handbook: a practical approach to planning and marketing. London: Continuum., 2000.

GOODWIN, H, Santilli, R. Community-Based Tourism: a success? ICRT Occasional Paper 11..,2009. Acessado em 18 de Jan. Disponível em:〈http://www.icrtourism.org/documents/OP11merged.pdf>

HAINSWORTH, D. Jamieson, W. A Toolkit for Monitoring and Managing Community-based Tourism, SNV Vietnam and Asia Pro-Poor Sustainable Tourism Network and University of Hawaii, Hanoi., 2007.

IFE, J. Community development: Community- based alternatives in an age of globalization., 2002.

INSTITUTO de Desenvolvimento Sustentável Mamirauá. (2011). Balanço Social 2001-2010. Acessado em 18 de Jan. Disponível em: http://mamiraua.org/cms/content/public/documents/c1231c347425-4e35-8337-f3445ef90a0_Balanço\%20Social\%20IDSM\%2024maio11\%20resolução\%20web.pdf.

JHA, S., Rao, V. \& Woolcock, M. Governance in the gullies: democratic responsiveness and community leadership in Delhi's slums. World Development 35(2), 230-246., 2007.

JONES, S. Community-based ecotourism the significance of social capital. Annals of Tourism Research 32:303-324., 2005.

JORDAN, J.; C. Hunt; \& A. Stronza. An ecotourism partnership in the Peruvian Amazon: the case of Posada Amazonas (Chapter 3: 30-48). In A. Stronza and W. Durham. Ecotourism and Conservation in the Americas. Cabi: Oxfordshire, Reino Unido, 2008.

KANE, M. \& Trochim, W. Concept Mapping for Planning and Evaluation. Thousand Oaks, CA: Sage Publications., 2007.

KIRK, P., \& Kraft, M. K. Community leadership development. Community Development Journal, 39(234-251)., 2004.

LAVERACK, G., \& Thangphet, S. Building community capacity for locally managed ecotourism in Northern Thailand. Community Development Journal, bsm058., 2007.

LEACH, M.; R. Mearns, e I. Scoones. Environmental entitlements: Dynamics and institutions in community-based natural resource management. World Development 27(2): 225-247., 1999.

MACLELlAN-Wright, F., Anderson, D., Barber, S., Smith, N., Cantin, B., Felix, R., et al. The development of measures of community capacity for community-based funding programs in Canada. Health Promot. Int., 22(4), 299-306., 2007.

MARIS, G. and Schipani, S. Monitoring Community based ecotourism in the Lao PDR: the UNESCONTA Lao Nam Ha ecotourism project monitoring protocol, UNESCO-National Tourism Authority of Lao PDR Nam Ha Ecotourism Project., 2001.

MENDOZA, E.; S. Perz, and C. Aguilar. The "Knowledge Exchange Train": a model for capacity building for participatory governance in the south-western Amazon. Development in Practice 17(6): 791-799., 2007 
MIELKE, E. J. C. Desenvolvimento Turístico de Base Comunitária. Campinas, São Paulo: Átomo \& Alínea, 2009, v.1. p.190., 2009.

MIELKE, E. J. C., Pereira. A. Desenvolvimento Econômico e Social através do Turismo: interações entre atores locais. In: Anais do IV Seminário de Pesquisa em Turismo do Mercosul / III Seminário da ANPTUR - Associação Nacional de Pesquisa e Pós graduação em Turismo, Caxias do Sul, Brazil., 2006.

MILLS, R. C. Sustainable community change: A new paradigm for leadership in community revitalization efforts. National Civic Review, 94, 9-16., 2005.

MOSCARDO, G. (Ed.). Building community capacity for tourism development. Australia , 2008.

MITCHELL, J. \& Muckosy P A misguided quest: Community-based tourism in Latin America ODI Opinion 102, 2008.

MORRA, L. \& Rist, R. The Road to Results: Designing and Conducting Effective Development Evaluations. Washington, DC: World Bank., 2009

PÁDUA, J.A. (Dois séculos de crítica ambiental no Brasil. Ciência Hoje 256 (156): 42-48., 1999.

PEGAS, F., \& Stronza, A. Ecotourism and sea turtle harvesting in a fishing village of Bahia, Brazil. Conservation and Society, 8(1), 15-25., 2010.

PEGAS, F., A. Coghland, e V. Rocha. An exploration of a mini-guide programme: training local children in sea turtle conservation and ecotourism in Brazil. Journal of Ecotourism. DOI:10.1080/14724049.2011.631710., 2011.

QUEIROZ, H. \& N. Peralta. "Reserva de Desenvolvimento Sustentável: Manejo integrado dos recursos naturais e gestão participativa" (pp. 447:476), 2006. Em Becker, B \& Garay, I. (eds.). Dimensões humanas da biodiversidade. Rio de Janeiro, Vozes. Acessado em 18 de Jan. Disponível em: $<\mathrm{http} / / / \mathrm{mamiraua} .0 \mathrm{rg} / \mathrm{cms} / \mathrm{content/public/documents/publicacao/93b463dd-b86a-4346-bda3-}$ b0ff95663c17_livro.2\%20QUEIROZ.pdf>.

REIDAR, D. Evaluating Development Programmes And Projects. Sage Publications. New Delhi, India., 2004.

RODRIGUEZ, A. Tourism, indigenous peoples and conservation in the Ecuadorian Amazon (pp. 155165). In A. Stronza and W. Durham. Ecotourism and Conservation in the Americas. Cabi: Oxfordshire, Reino Unido.,2008..

SANTINI, R. \& Goodwin, H. (2009) Community-based tourism: a success? International Centre for Responsible Tourism ICRT Occasional Paper 11., 2009. Acessado em 18 de Jan. Disponível em: <www.icrtourism.info>.

SAXENA, G. Beyond mistrust and competition - the role of social and personal bonding processes in sustaining livelihoods of rural tourism businesses: a case of the Peak District National Park. International Journal of Tourism Research 8: 263-277., 2006.

SCHEYVENS, R. Exploring the Tourism-Poverty Nexus. In: M. Hall, ed(s). Pro-poor Tourism: Who Benefits? Clevedon: Channel View Publication., 2007.

SEBOLA, M., \& Fourie, L. Community participation in ecotourism destinations: Maleboho Nature Reserve. Transactions on ecology and the environment, 197., 2006.

SNV \& UH-University of Hawaii A Toolkit for Monitoring and Managing Community-Based Tourism. SNV Asia Pro-Poor Sustainable Tourism Network; SNV Vietnam; University of Hawaii., 2007. Acessado em 18 de Jan. Disponível em: <http:// http://www.snvworld.org/en/Documents/Knowledge\%20Publications/A\%20toolkit\%20for\%20monitor ing\%20and\%20managing\%20community-based\%20tourism.pdf. >. 
SPENCELEY, A. Responsible Tourism: Critical issues for Conservation and Development. Earthscan, London., 2008.

SPRECHER, D. \& Jamieson, D. A Manual for Monitoring Community Tourism Development. Canadian Universities Consortium, Thailand., 2000.

STRONZA, A., and J. Gordillo. Community Views of Ecotourism: Redefining Benefits. Annals of Tourism Research 35(2):444-468., 2008.

STRONZA, A., and Pegas, F. Ecotourism and Conservation: Two Cases from Peru and Brazil Human Dimensions of Wildlife 13:263-279., 2008.

The Mountain Institute. Community-based Tourism for Conservation and Development: A Resource Kit, 2000. Acessado em 15 de Jan. Disponível em http://www.mountain.org/docs/TBC-Kit-final2003.pdf

The Global Fund Monitoring and Evaluation Concepts, 2010 Acessado em 03 de Jan. Disponível em: http://www.ilfondoglobale.org/documents/me/ME_Concepts.pdf>

Trejos, B. e L. Chiang. Local economic linkages to community-based tourism in rural Costa Rica. Singapore Journal of Tropical Geography 30: 373-387, 2009.

UNDP. Human Development Report 2006 Beyond Scarcity: Power, Poverty and the Globar Water Crisis, United Nations Development Programme Energy and Environment Group, New York, EUA. 2006

UNWTO. Signposts for Sustainable Tourism: A Guidebook for The Development and Use of Indicators for The Development of Tourism Destinations. United Nations World Tourism Organization. Madrid, Espanha, 2004.

Poverty Alleviation Through Tourism - A Compilation of Good Practices. United Nations World Tourism Organization. Madrid, Espanha, 2006

VELLASs, F. Monitoring the costs of and benefits of ecotourism: equitable distribution between all members, Discussion paper for the World Ecotourism Summit, UNWTO, Madrid, 2002.

WEARING, S., \& McDonald, M.. The development of community-based tourism: Re-thinking the relationship between tour operators and development agents as intermediaries in rural and isolated area communities. Journal of Sustainable Tourism, 10 (3), 191-206, 2002.

WILLIAMS, M. R., \& Wade, V. M. Sponsorship of community leadership development programs: What constitutes an ideal partnership? Journal of the Community Development Society, 33(61-71), 2002.

WILSON, S, Fesenmaier, J., D. Fesenmaier, \& J. C. van Es. Factors for Success in Rural Tourism Development. Journal of Travel Research, v. 40, Novembro. p.132 - 138., 2001.

WORLD Bank. Understanding and setting up monitoring and evaluation system., 2006. Acessado em 19 jan. Disponível em http://web.worldbank.org/WBSITE/EXTERNAL/TOPICS/EXTPOVERTY/EXTISPMA/0,,menuPK:3 84336 pagePK:149018 piPK:149093 theSitePK:384329,00.html

WWF International (2001) Guidelines for community-based ecotourism development WWF International, 2001. Acessado em 17 de jan. Disponível em: http://www.icrtourism.org/Publications/WWF1eng.pdf.

\section{Recebido em: 01/02/2012}

Aprovado em: 14/06/2012 